\title{
Claudel lecteur de Saint-John Perse
}

\author{
Glenn Fetzer \\ Calvin College
}

L'idée de relire Saint-John Perse à travers le regard de Paul Claudel s'impose s'il s'agit de mesurer la portée de Claudel esthéticien et si l'une des preuves de la critique de la poésie réside dans la cohérence entre les principes épousés et leur mise en pratique. D'autant plus si dans ses essais sur la critique Claudel a tendance à développer une appréciation de la poésie en parallèle à celle de l'art. À part les différences qu'on peut discerner entre la critique de l'art et celle de la poésie, dans le cadre de sa critique il reste un autre écart important : celui de la distance temporelle et des rapports personnels. Le critique entreprend-il son appréciation d'un poète d'un siècle antérieur de la même manière qu'il le ferait s'il s'agissait d'un poète contemporain, voire un ami ? Là est la question d'où naît une critique équivoque car dans son étude sur « Vents » de Saint-John Perse, Claudel met en jeu ses propres apports sur la critique de l'art.

Le premier axe de l'équivoque se lève dans le contexte des rapports personnels et professionnels entre les deux poètes. De tels rapports rendent le travail critique plus difficile comme reconnaît Claudel le 29 juin 1949 dans une lettre à Saint-John Perse, où il prévoit entreprendre bientôt un essai sur "Vents ». "Loin d'aider ", écrit-il, "l'intimité personnelle qu'on a avec un auteur est pour l'appréciation un obstacle dont il faut se dégager ${ }^{1}$. L' L $^{\prime}$ obstacle $»$ qu' avoue Claudel par cette mise en garde remonte bien des années et court le risque de brouiller depuis longtemps l'acuité de sa vue critique. Comme témoignent les lettres entre les deux, ainsi que la correspondance de Claudel, Francis Jammes, et Gabriel Frizeau, Claudel et Saint-John Perse se connaissent depuis 1905, à l'époque où celui-ci n'avait que dix-huit ans et était étudiant en droit à Bordeaux. Dès ce moment un rapport entre les deux écrivains s'est établi, dont les traits saillants se sont avérés résistants à tout changement important. Si le respect, la discrétion, et l'admiration figurent parmi les caractéristiques principales dont témoigne Alexis Léger à l'égard de son aîné, celui-ci, en retour, va maintenir à l'égard de son plus jeune 


\section{Glenn Fetzer}

collègue un rapport fondé sur une sollicitude amicale et des conseils, mais aussi sur un respect profond.

Dans le rapport entre les deux hommes, c'était Claudel qui avait pris l'initiative. Les premières lettres d'Alexis Léger au diplomate établi et l'écrivain témoignent de l'embarras devant l'attention soutenue à son égard $^{2}$. À partir de juin 1907, au moment où le jeune poète, vulnérable, orphelin récemment de père, et cherchant sa voie dans le monde, s'est permis d'écrire à Claudel, c'est évident que les relations entre les deux allaient se dérouler, avant tout, au niveau des conseils professionnels. En 1911 déjà, à l'insistance des amis à lui, Claudel est intervenu en faveur d'Alexis Léger pour faciliter son entrée dans la carrière consulaire ${ }^{3}$. La profondeur de reconnaissance qu'a témoignée maintes fois Alexis Léger envers son guide en matière de carrière et en écriture a persisté pendant les années de ses affectations successives en service diplomatique et a continué pendant les années de son exil ${ }^{4}$.

$\mathrm{Si}$ les lettres personnelles qu'ont échangées les deux diplomates aux années 1920 et 1930 rendent témoignage à une amitié nourrie par un vif intérêt commun aux lettres et à la vie de l'esprit, c'est par le biais d'engagements politiques que le septuagénaire Claudel lit d'emblée SaintJohn Perse lors de la publication de ses poèmes aux années 1940 mais surtout lors de la parution de "Vents" en 1946. Entre 1945 et 1949 on s'aperçoit de la part de Claudel un changement subtil dans son appréciation de Saint-John Perse, poète. Bien que son jugement initial de cette époque - constaté dans une lettre du 12 avril 1945 - reconnaisse les quatre poèmes qu'il venait de recevoir ("Exil », "Pluies ", " Neiges », et "Poèmes à l'étrangère ") comme " magnifiques ${ }^{5}$, il voit avant tout en Saint-John Perse la perte d'un collègue estimé. En effet la référence élogieuse des poèmes se fait en passant, ouvrant la voie à Claudel de regretter l'absence de son ami : « Le malheur a du moins révélé à la France le grand Poète qui se cachait sous le Serviteur méconnu » (HSJP 439). Et puis, après quelques remarques supplémentaires au sujet des poèmes, il reprend d'un ton nostalgique l'absence tant regrettée : "Aurons-nous la joie de vous revoir bientôt en France? » se demande-t-il? Vos amis ne vous ont pas oublié et désirent que vous remettiez au service du pays l'intelligence inestimable que vous êtes " (HSJP 440).

Pas tout à fait deux ans plus tard, Claudel a l'occasion de s'exprimer de nouveau. Il s'agit cette fois de la publication de "Vents " en 1946, devant laquelle Claudel s'annonce pris de " respect " et d' «admiration » (HSJP 463). D'ailleurs, le contenu de la lettre (qui date du 
20 février 1947) laisse voir une prise de conscience profonde de l'art du poète sans pour autant s'attarder trop sur Alexis Léger, diplomate regretté. Les quelques lignes de cette brève communication témoignent d'une nouvelle perspective à travers laquelle Claudel commence à regarder son ami : celle d'un grand poète.

Plus il se trouve face aux poèmes persiens de cette époque plus Claudel devient un lecteur attentif. Quand, en 1949, il écrit de nouveau à Saint-John Perse après s'être décidé à lui consacrer un article, c'est d'un élan d'enthousiasme jusqu'alors inconnu. Le passage d'un lecteur plutôt inexpressif et détaché à l'un qui est ouvertement admirateur se lit dès l'incipit de la lettre du 29 juin 1949 : « Je me sens devant votre œuvre, » écrit-il, « comme devant quelque chose d'important et que l'on n'aborde pas à la légère » (HSJP 463). Nous nous apercevons bien chez Claudel une estimation pour Saint-John Perse qui ne cesse d'augmenter. De la reconnaissance, en 1945, de quatre poèmes magnifiques, et de l'aveu — deux ans plus tard - d'un respect et d'une admiration pour « un très grand poète " (HSJP 463), Claudel répand sa louange à un niveau plus large - jusqu'à l'œuvre elle-même. À peine un mois plus tard, dans une lettre du 25 juillet 1949 , et après avoir achevé son étude sur "Vents », Claudel reste ferme dans son appréciation de son plus jeune collègue : «Quel grand poète vous êtes devenu ", écrit-il (HSJP 464). Le ton de ses lettres de 1949 reflète l'estime que le poète aîné porte vers l'œuvre de son ami.

Cependant, au cours des lettres des années 1940, culminant avec celles au sujet de "Vents", le rapport entre les deux poètes reste hiérarchique. Aussi grand un poète qu'est devenu Saint-John Perse et aussi important que s'avère son œuvre, il est évident que la renommée dont il se jouit tient de Claudel, qui le constate et le lui fait savoir. Le 29 juin 1949, avant d'entamer son étude, Claudel annonce à son collègue l'intention de " prendre les dimensions du poème ("Vents ») et d'en faire un " article très étudié " qui servira d'établir le poète " en pleine lumière à [son] rang » (HSJP 463). À peine un mois plus tard (le 25 juillet), il lui fait savoir également la filière choisie pour réaliser cette renommée : « Je destine cette ceuvre importante ", lui écrit Claudel, " à la Revue de Paris " (HSJP 464).

Mais malgré l'enthousiasme de Claudel, il se garde de s'en laisser emporter. La phrase qui suit inscrit cet enthousiasme dans un devoir qui date de loin et que Claudel terminera avant de passer à l'étude sur SaintJohn Perse : l'achèvement d'un commentaire d'Isaïe. On ne peut que remarquer l'ironie de ce détail. La louange dont témoigne Claudel devant l'œuvre se tempère du rappel à son ami de l'écart fondamental qui les 


\section{Glenn Fetzer}

distingue : la religion ${ }^{6}$. C'est un écart auquel Claudel fait référence et qui servira d'éclaircir sa réticence d'écrire sur "Vents ». Au delà l'obstacle que "l'intimité personnelle " lui représente, on s'aperçoit de cet autre écart dans la lettre du 29 juin 1949 : « Et du fait de votre agnosticisme total, mon cher Léger, vous êtes si loin de moi! » (HSJP 463). En matière de religion, ainsi qu'en tant qu'artiste, Claudel ne peut pas s'empêcher de se voir le modèle selon lequel on fait la mesure, ou dans ce cas, de se voir celui de qui on s'éloigne. Et c'est ainsi que dans l'étude sur "Vents ", Claudel a tendance à évaluer l'œuvre à travers l'homme, et surtout, à travers l'Alexis Léger connu en diplomate et vieil ami mais aussi en agnostique.

Cependant; la lecture de "Vents " témoigne également de l'éloignement de la part de Claudel de son rôle principal d'exégète. C'està-dire que si l'équivoque discernée chez le critique s'élève dans le cadre des rapports personnels et professionnels, elle se prolonge dans le contexte de la priorité qu'accorde le critique à la compréhension. Comme remarque Michel Lioure, la question claudelienne par excellence devant une œuvre d'art s'avère une question de l'herméneutique : «Que veut dire cette œuvre? ${ }^{7}$ » Lioure résume ses propos ainsi :

Claudel applique donc en critique d'art ses méthodes exégétiques. Épris de symbolisme et de spiritualité, il " écoute " les tableaux comme il interroge l'Apocalypse ou le Cantique des Cantiques. Ses interprétations sont bien des " exégèses ", obéissant aux mêmes principes et aux mêmes partis que ses travaux bibliques. Partout il subordonne ou sacrifie le signifiant au signifié, le sens littéral au sens figuré, la lettre à l'esprit, l'image au symbole. (32)

Pour sa part, Claudel élabore le lien entre l'exégèse et la religion dans son essai « Religion et poésie " $(1928)^{8}$, où il introduit le rôle de l'exégète dans une compréhension particulière de l'univers. Cette vision du monde (ou «l'univers de Dieu », comme il en parle) s'appuie sur les Crédos de la religion chrétienne. Il s'agit du principe d'un monde composé des choses visibles et choses invisibles (142). Celles-là sont instruites par la raison, l'imagination, et les sens et forment le domaine de la science. Celles-ci sont instruites par la raison et la foi et constituent le domaine de l'art et de la poésie. D'après Claudel la connaissance d'une chose suppose l'acte d'embrasser par la pensée son existence. Tel est le 
propre de la science. La compréhension d'une chose, par contre, entraîne une connaissance de sa construction, ses relations, et son origine (143).

Que ce soit par rapport à la critique de la poésie ou celle de l'art plastique, la vocation critique de Claudel, comme souligne Lioure, se fond sur l'analyse et sur l'interprétation (13). Selon Lioure, le regard critique de Claudel dépasse une simple description des choses, car la description «n'est pas destinée à suppléer la vision mais à illustrer la réflexion » (22). De plus, en prenant la mesure d'une ouvre, il a tendance à y noter « un centre, un centre de gravité » qui lui permet de proposer le sens de l'œuvre (23).

Quelle devrait être l'attitude du critique face à l'œuvre du peintre ou du poète? Claudel fournit une réponse qui engage par nécessité une prescription herméneutique :

Il faut que le témoin de l'artiste, que le lecteur du poète puisse dire en l'écoutant: Oui, voilà ce que j'aurais voulu dire moimême, voilà l'idée que j'aurais voulu mettre au jour moi-même pour savoir ce qu'elle a à faire et à dire ${ }^{9} .$.

Ce passage est révélateur en ce qu'il fait comprendre un principe concernant la poésie que Claudel exprime ailleurs : le souhait - ou même l'attente - que le poème comporte des idées. Ou au moins, le poème comme porteur d'idées s'avère un principe auquel tient Claudel poète. « Je considère les formes dramatiques ou poétiques ", écrit-il, "comme des moyens qui conviennent à exprimer certaines de mes pensées $»^{10}$.

Étant donné la prédilection de Claudel pour une critique exégétique et la préférence de se voir à la place du poète, plusieurs questions peuvent se poser. En lisant «Vents », Claudel penche-t-il pour une lecture avant tout exégétique? Se contente-t-il de suborner le signifiant au signifié? Et, en tant que lecteur du poème, s'exclame-t-il, «Oui voilà ce que j'aurais voulu dire moi-même, voilà l'idée que j'aurais voulu mettre au jour? » Sans nous occuper de la dimension des rapports personnels entre les poètes, lesquels nous avons déjà abordé et qui semblent exercer une influence considérable sur la critique, suffit-il de constater que dans l'essai sur « Vents ", Claudel dément ses principes de critique. Ou au moins, l'expérience s'avère infirmer ces propositions.

Dès l'incipit de l'essai, le critique reconnaît une poésie différente de celle qu'il a l'habitude de commenter. "Vents " fait partie de cette poésie qui se prête à une lecture tout autre que celle qui s'appuie sur 


\section{Glenn Fetzer}

l'interprétation des signes. "Il y a une poésie nourrie par l'espace ", débute-t-il, " et à qui il faut de l'espace pour se développer, une poésie qui naît moins de l'ajustage précis d'une combinaison verbale que de l'attention hors de nous à un ensemble $"^{11}$. L'ensemble à qui il fait référence est celui du genre épique et, comme il fait savoir au poète vers la fin de juillet 1949, le premier point de son essai est que «Vents» est un poème épique » (HSJP 464). Or, si, selon Claudel, l'épopée — que ce soit le récit d'un roman ou d'un poème - se distingue par des éléments constituent, reliés en quelque sorte, «non seulement par les lois d'une causalité dynamique ou morale, mais par celles de l'équilibre et d'une parenté secrète ${ }^{12}$, alors la fonction attribuée à la signification se déclare d'emblée beaucoup moindre.

Le reste de l'essai témoigne d'une préférence pour l'image évoquée plutôt qu'expliquée. En s'absentant largement de l'interprétation, Claudel se borne à soulever des images au choix et à les commenter. En même temps il nous renseigne sur ses propres démarches et sur celles du poète qu'il lit. Ainsi, nous dit-il,

Mais le propos du poète qui nous invite ici à l'accompagner est bien différent du mien. Il ne s'agit pas de comprendre, mais de deviner, dans le sens étymologique du mot : divinari. Il ne s'agit pas tant d'une intelligence analytique, résultant d'une étude extérieure de la forme, des organes, de la politique, de l'histoire, de la pensée, que d'une intelligence intérieure non accompagnée d'assentiment. (HSJP 47)

Comment souligner les divergences des démarches poétiques d'une manière plus nette? Claudel ne peut pas s'expliquer avec plus de clarté. Mais son propos joue-t-il un rôle secondaire? Se peut-il que le critique est en train de justifier son approche à lire Saint-John Perse? Qu'il se met à l'abri de ceux qui attendraient de lui une certaine façon de s'y prendre? Quoi qu'il en soit, Claudel revendique sa stratégie de lecture. Loin de nier l'importance du sens, il met l'accent sur le ton : "le sens de ce qu'on nous dit est souvent moins important que le ton " (HSJP 47). D'ailleurs, prétend-il, «Tout veut dire, mais rien ne signifie qu'en excluant la traduction, suivant cette mésintelligence essentielle dont Saint-John Perse nous entretenait » (HSJP 47). Si le critique se passe d'une lecture de compréhension, toute la charge en retombe sur Saint-John Perse. 


\section{Claudel lecteur de Saint-John Perse}

C'est toujours au sujet de la signification de «Vents » que Claudel soulève ce qu'il appelle les "obsessions » du poème, dont la première serait l'omniprésence du texte (HSJP 48) et la deuxième, la place accordée à "l'intersigne ", au " chiffre indéchiffrable ", à " la chose qui veut dire " (HSJP 48). S'appuyant sur une lettre où Saint-John Perse rappelle quelques impressions insolites et singulières de ses visites dans tous les musées d'Europe, Claudel commente la nature fragmentaire de cet assemblage d'images et de mots. Pour lui, l'accent sur les signifiants, sur « les vocables hétéroclites » venus d'il ne sait où, sert moins à signifier qu'à catalyser (HSIP 49). C'est ainsi que la lecture de Claudel résiste à toute tentative de réduction dans le domaine de la signification et se contente de déceler quelques-uns des liens de cette "parenté secrète " propres au genre épique.

Reste la question de la cohérence des deux éléments. Face au poème, Claudel admet-il à une envie de faire pareil? Sa lecture de "Vents ", déclenche-t-elle chez lui une réponse d'assentiment, une expression d'approbation enthousiaste? La réponse reste évidente. Comme nous avons déjà remarqué, Claudel ne s'épargne pas d'efforts à distinguer son propos de celui de Saint-John Perse (HSJP 47). Si pour lui, il est question de comprendre, pour le poète du "Vents » il s'agit plutôt de deviner (HSJP 47). Au fond pour Claudel, puisque la compréhension ne peut se concevoir à part du contexte religieux, toute réponse du critique remonte à la religion. Et, comme Claudel nous fait rappeler, « Dieu est un mot que Saint-John Perse évite [...] religieusement » (HSJP 52).

C'est justement le côté religieux des choses, en dernier lieu, qui constitue le troisième axe de l'équivoque dans l'étude sur « Vents ». Dans le texte " Religion et poésie » (1928) — le même essai dans lequel Claudel précise les liens entre l'exégèse et la religion, les choses visibles et les choses invisibles, et la connaissance et la compréhension — il constate, même, les enjeux d'une poésie sans compréhension. Il présume, en fait, que sans une compréhension avouée du monde, un poète (n'importe lequel) fera défaut. " Bien sûr, » écrit-il, « même sans une idée générale de la terre et du ciel, vous pouvez faire de la très jolie poésie, vous pouvez ciseler de délicates œuvres d'art, vous pouvez combiner des bibelots fort curieux et intéressants » (143). Bien qu'une poésie dénuée de compréhension (dans la manière dont Claudel envisage la compréhension) réussisse à plaire ou, autrement, à faire une grande impression, elle ne jouira pas de l'approbation de Claudel. D'ailleurs, l'œeuvre d'un tel poète, prétend-il, est païenne : « Mais dans cette poésie païenne, il y a toujours à mon avis 


\section{Glenn Fetzer}

quelque chose d'étriqué et de gêné. Même pour le simple envoi d'un papillon », poursuit-il, « le ciel tout entier est nécessaire » (143).

Pour Saint-John Perse, paraît-il, les jeux sont faits. Sa poésie est païenne, d'après ce que Claudel avait écrit plus de vingt ans avant l'essai sur «Vents ». Et le critique tient à cette position lors de son étude. Dans sa lettre du 9 août 1949, Claudel résume pour Saint-John Perse les deux points principaux de son étude, dont le premier nous avons déjà considéré ( "Vents" est un poème épique »[HSJP 464]). Mais c'est le deuxième point qui s'impose : "Saint-John Perse est un païen ou plutôt un préchrétien, pour qui l'expectatio creaturae n'a eu ni satisfaction ni commencement de satisfaction » (HSJP 46). Alors, on est à croire, donc, que dans le poème "Vents » il y a " quelque chose » d'étriqué et de gêné ". Après tout, dans l'étude sur "Vents », ne s'est-il pas donné beaucoup de mal pour se distancier de la démarche poétique de Saint-John Perse? Cependant, toute la portée de l'essai semble récuser l'emprise de l'étriqué dans le poème. Car, dès le début, Claudel y voit un désir de l'espace et une attention vers ce qui est en dehors de nous (HSJP 43).

Or, une poésie "sans Dieu " — comme serait celle de Saint-John Perse - comporte plusieurs caractéristiques ou, comme le précise Claudel dans son essai sur la religion et la poésie, des "ingrédient[s] essentiel[s] ", qui manquent. Parmi ces motifs dont sont dépourvus les poètes païens, ou ces "immenses avantages que la religion apporte ", Claudel en profère trois : la louange, le sens, et le drame (148-149). Par le premier avantage — la louange - il entend « l'expression du besoin le plus profond de l'âme, la voix de la joie et de la vie " (148), le sens inébranlable de la présence d'autrui (148). Quant au deuxième - le " sens" - il faut le comprendre selon ses implications téléologiques. Selon Claudel, "sens " désigne un système de rapports dont Dieu est à l'origine et dont il constitue la fin (148). Le troisième avantage qu'apporte la religion - le Drame - s'inscrit, lui aussi, dans le cadre de la "Révélation Chrétienne » (148), et se rapporte avant tout à la morale et aux actes humains. Le Drame comprend tout le possible dans la vie de l'individu en poursuivant la destinée humaine.

Certes, Saint-John Perse ne dispose pas de la foi chrétienne. Et qui plus est, si l'on accepte les critères claudeliens des ingrédients essentiels qui manquent aux poètes non-croyants, il s'ensuit que Claudel a bien raison de lire Saint-John Perse comme poète païen. Cependant, on se permet de soulever quelques questions. "Vents " est-il un poème dépourvu de louange? De sens? De drame? Ou peut-être plus pertinent : 
dans son étude de "Vents » Claudel reconnaît-il des moments de louange, de sens, ou de drame?

À l'un côté les propos de Claudel se justifient. Cette justification se développe lors de son constat que "Vents » est un poème épique. Le concept du poème épique est éclairci dans son essai « La Poésie est un art » (17), où ses précisions sur le poème épique se juxtaposent à celles du poème dramatique. Tandis que le poème dramatique se connaît par une action confiée aux acteurs (17), le poème épique est un « spectacle » qui s'avère contribuer à l'espace où se déroulera plus tard l'action dramatique. Claudel l'explique de cette manière :

Le poème épique est un spectacle, le poème dramatique est une action. Une action confiée à des acteurs. Comme le poème épique développe une situation, c'est-à-dire une espèce de site moral, le drame actualise, complète, authentifie, élève à la valeur d'exemple, un de ces débats enchoatifs, plus ou moins riches de signification, au milieu desquels la vie courante ne cesse de nous promener. " (17)

Selon les distinctions ainsi esquissées, on ne peut qu'accepter l'absence du drame dans un poème épique. Et, en tant que conséquence logique, il survient que le poème de Saint-John Perse ne jouit pas de ce troisième avantage qu'apporte la religion.

Mais juger "Vents » sur les apparences ainsi présentées par Claudel est de présumer la question résolue. Chez Saint-John Perse, n'a-ton pas raison de voir dans les évocations des peuples en quête de nouveaux enjeux humains l'empreinte de la louange? Quand le poète écrit,

Mais c'est de l'homme qu'il s'agit! Et de l'homme luimême quand donc sera-t-il question? - Quelqu'un au monde élèvera-t-il la voix?

Car c'est de l'homme qu'il s'agit, dans sa présence humaine; et d'un agrandissement de l'œil aux plus hautes mers intérieures.

Se hâter! Se hâter! témoignage pour l'homme ${ }^{13}$ ! 
ne témoigne-t-il pas de la joie, du besoin profond d'unité, de la vie dans la plénitude et l'étendue de son expression? Et Claudel qui lit le même chant et qui cite des brins d'énoncés du même passage, pourquoi n'admet-il pas ce sens de louange? En outre, pourquoi se contente-t-il d'amonceler les fragments de vers de sorte qu'on n'y lise que d'une soif à jamais assouvie, si ce n'est que pour soutenir la prétention, faite déjà une quinzaine de jours avant d'entamer l'étude, que l'agnosticisme de SaintJohn Perse sert à l'éloigner plus que jamais du critique? Par ailleurs, si "l'intimité personnelle " avec l'auteur, dont parle Claudel en juin 1949, risque être un obstacle, n'est-il pas possible que Claudel ne réussisse pas (pour quelque raison qu'il soit) à le surmonter?

Malgré la sévérité par laquelle Claudel met fin à son étude, il laisse entrer une ambiguïté qui adoucit la critique et qui nous donne lieu à remettre en question le silence de Claudel par rapport aux propos de Saint-John Perse sur la spiritualité. Il s'agit du jugement final de "Vents » où il s'en prend à Saint John Perse pour ne pas s'être servi du mot «Dieu » dans ses poèmes. "Mais Dieu ", a-t-il écrit, " est un mot que Saint-John Perse évite, dirai-je religieusement? Et que pour un empire il ne laisserait pas sortir de ses lèvres » ${ }^{14}$. Implicitement, l'absence du mot « Dieu, » selon Claudel, ne s'élève d'une insouciance quelconque ni d'une indifférence, mais plutôt d'un choix délibéré. Et c'est à ce refus prétendu du mot que Claudel s'attaque, sans pour autant voir dans la poésie le trait du spirituel.

Néanmoins, dans cette critique de Saint-John Perse, l'emploi figuratif du mot " religieusement » fait voir un sens double. Par cette équivoque, Claudel nous permet de considérer - ne fût-ce que pour un instant - la présence du sacré chez Saint-John Perse que Claudel passe sous silence. À l'encontre d'une expression religieuse claudelienne qui se connaît par propositions, la place du spirituel chez Saint-John Perse s'effectue dans la langue et dans le poein. Comme l'indique Yves-Alain Favre, c'est dans la langue où Saint-John Perse s'efforce d'instaurer du cérémonial et du sacré ${ }^{15}$. Le rituel du langage où tout est solennisé, la structure des versets et la composition orchestrée du poème témoignent, tous, d'un sacré primitif (49). Selon Favre, la poésie persienne " est un exercice spirituel qui ne dépend pas de l'objet de la contemplation [...] mais au sujet qui contemple " (54). Par conséquent dans cette poésie, et surtout dans «Vents » où l'on est conscient de l'espace, de l'ouverture, et de l'infini, la présence percevante s'engage dans une entreprise sacrale, et sacralisante. À la critique de Claudel que seule la religion peut apporter une compréhension de l'universel ("Religion et poésie »142), et face à sa 
conscience dans «Vents » d'une « poésie nourrie par l'espace » qui « naît moins de l'ajustage précis d'une combinaison verbale que de l'attention hors de nous à un ensemble " (Un poème de SJP 43), Favre aurait pu lui signaler que chez le poète "le sacré n'est attaché à aucun lien précis ", que "Tout l'univers se trouve sacralisé ", que "les deux catégories antithétiques du profane et du sacré ne s'y opposent plus », mais qu' « en tout lieu le poète éprouve ce "mysterium tremendum" " (Favre 64). Si dans sa lettre du 9 avril 1949, Claudel annonce que l'un des deux « points essentiels » de son essai est que "Saint-John Perse est un païen ou plutôt un préchrétien " (HSJP 464), il néglige de développer ce " point essentiel ", ou même, de l'expliciter dans son étude.

Aussi important que l'essai de Claudel s'est révélé en faisant la renommée du poète, s'agit-il plutôt de l'auteur de l'essai que d'« Un Poème de Saint-John Perse » ? À travers l'étude, Claudel ne se laisse-t-il pas porter vers une lecture intéressée qui s'est influencée autant par un prosélytisme immodéré et par sa connaissance de longue date d'Alexis Léger que par ses principes de critiques bien définis? Certes, lire «Vents » oblige Claudel à se faire face à une conception de la poésie différente de la sienne. C'est une différence dont il a pu prendre la mesure mais qui a encore accentué la réticence de la part du critique à appliquer avec rigueur ses méthodes exégétiques. Si Claudel fait entrer dans cette étude un peu moins d'exégèse et un peu plus de critique personnelles, il court le risque d'une interprétation hasardeuse où l'on sent trop la main de l'auteur. En fin de compte, peut-être, est-ce là le plus grand mérite de l'étude dont l'équivoque et la vision subjective d'un poème et d'un poète moderne engage les lecteurs à lire et poètes et leurs critiques d'un œil neuf.

\section{Notes}

1 Honneur à Saint-John Perse, hommages et témoignages littéraires suivis d'une documentation sur Alexis Léger (Paris : Gallimard, 1965) 463. Tous les renvois dans l'article présent à cet ouvrage, désormais abrégé HSJP, sont indiqués dans le corps de l'article.

2 Dans l'Euvres complètes, la première lettre à Claudel dont nous disposons date du novembre 1906 et laisse voir une certaine maladresse face à une lettre antérieure de Claudel : « Je vous remercie de votre lettre [...]. Il y a longtemps déjà. - Je ne vous écrivais pas, parce que je n'ai rien à vous dire. Je ne puis 


\section{Glenn Fetzer}

rien avoir à vous dire... Votre bonté m'est étrangère - et je ne possède pas avec vous un petit morceau de vie banale en commun, de la vie de chaque jour, qui lie seule » $(O C, 711-712)$.

3 Voir les pages de correspondance de Paul Claudel, Francis Jammes, et Gabriel Frizeau, reprise dans Honneur à Saint-John Perse, 606-09.

4 Par exemple, dans une lettre datée 15 fév 1910, Alexis Léger exprime un sens de reconnaissance dès le début : « Je vous suis tout reconnaissant. Frizeau m’a communiqué votre lettre. Je vous remercie. Je ne serai pas confus de vous avoir occupé de moi » (OC, 717-718).

5 Lettre à Saint-John Perse. Le 12 avril 1945, reprise dans HSJP (439).

6 La reconnaissance mutuelle de cet écart qui les séparait date, au moins, depuis 1907. Dans une lettre de juin de cette année-là, Saint-John Perse défend son point de vue sur la vie en désignant Claudel par la qualification de " chrétien ". Au cours des ans, Claudel a soulevé à plusieurs reprises la religion (ou son manque) comme marque de dissemblance entre les deux amis.

7 "Claudel et la critique d'art " dans La Revue des lettres modernes no. 12. "Claudel et l'art », sous la direction de Jacques Petit, nos. 510-515 (1977), p. 17. Tous les renvois à cet essai seront identifiés dans le corps de l'article par une référence à Lioure. Le sujet de l'étude de Lioure se limite à la critique de la peinture. Néanmoins, comme indiqueraient d'autres essais de Claudel, la critique de la poésie jouit des critères de jugement semblables.

8 « Religion et poésie » In Euvres complètes de Paul Claudel. Tome Quinzième, positions et propositions. (Paris : Gallimard, 1959), 141-149.

9 Paul Claudel, «Opinion pour l'éther. » Euvres complètes de Paul Claudel. Tome Dix-huitième. Accompagnements Discours et Remerciements (Paris : Gallimard, 1961), 13.

10 « Le rôle du poète dans la cité ». CEuvres complètes de Paul Claudel. Tome Dixhuitième. Accompagnements Discours et remerciements (Paris : Gallimard, 1961), 11.

11 Paul Claudel, "Un poème de Saint-John Perse : "Vents" ", rpt. In HSJP, 43.

12 Paul Claudel, "La poésie est un art ", CEuvres complètes de Paul Claudel. Tome Dix-huitième. Accompagnements, Discours et remerciements, 17.

13 Saint-John Perse, Euvres complètes (Paris : Gallimard, 1972), 224.

14 "Un poème de Saint-John Perse : "Vents" ", in HSJP, 52. Dans une autre version du manuscrit, celle qui paraît dans Accompagnements sous le titre "Un Poème de Saint-John Perse », la phrase se lit ainsi : " Mais Dieu est un 


\section{Claudel lecteur de Saint-John Perse}

mot que Saint-John Perse évite, dirai-je, religieusement? Et que pour un empire, n'est-ce pas Léger? Il ne laisserait pas sortir de ses lèvres " (239).

15 Yves-Alain Favre. Saint-John Perse : Le Langage et le sacré (Paris : José Corti, 1977) 48. 